This item was submitted to Loughborough's Research Repository by the author.

Items in Figshare are protected by copyright, with all rights reserved, unless otherwise indicated.

\title{
Model based control of a liquid swelling constrained batch reactor subject to recipe uncertainties
}

PLEASE CITE THE PUBLISHED VERSION

PUBLISHER

(C) Elsevier

VERSION

AM (Accepted Manuscript)

LICENCE

CC BY-NC-ND 4.0

\section{REPOSITORY RECORD}

Simon, Levente L., Zoltan K. Nagy, and Konrad Hungerbuhler. 2009. "Model Based Control of a Liquid Swelling Constrained Batch Reactor Subject to Recipe Uncertainties". figshare.

https://hdl.handle.net/2134/5652. 
This item was submitted to Loughborough's Institutional Repository (https://dspace.lboro.ac.uk/) by the author and is made available under the following Creative Commons Licence conditions.

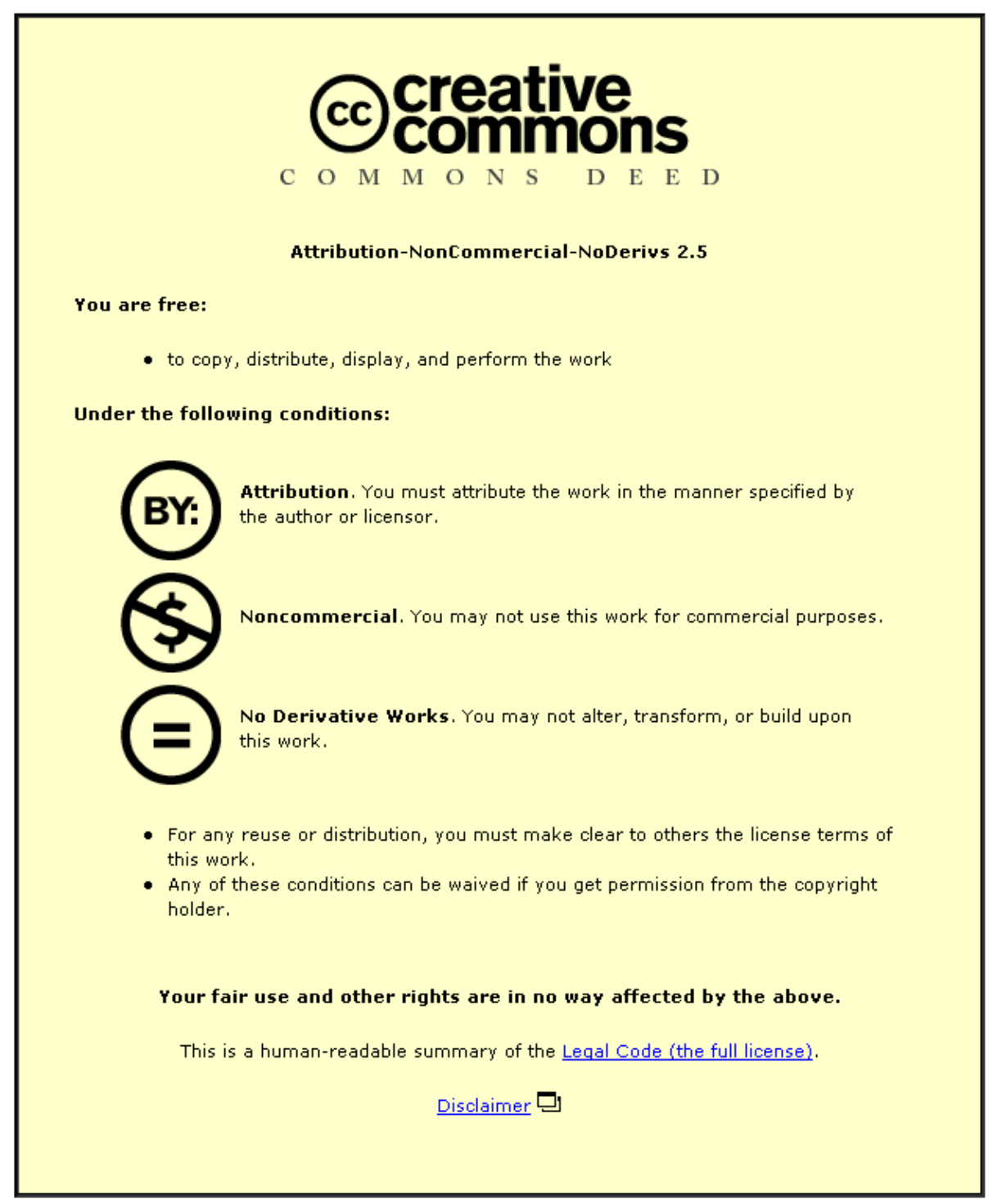

For the full text of this licence, please go to: http://creativecommons.org/licenses/by-nc-nd/2.5/ 


\title{
Model based control of a liquid swelling constrained batch reactor subject to recipe uncertainties
}

\author{
Levente L. Simon ${ }^{* 1+t}$, Zoltan K. Nagy ${ }^{\#}$, Konrad Hungerbuhler ${ }^{+}$ \\ ETH Zurich, Institute of Chemical and Bioengineering, Switzerland \\ ${ }^{\#}$ Loughborough University, Chemical Engineering Department, Loughborough, LE11 3TU, \\ United Kingdom
}

\begin{abstract}
This work presents the application of nonlinear model predictive control (NMPC) to a simulated industrial batch reactor subject to safety constraint due to reactor level swelling, which can occur with relatively fast dynamics. Uncertainties in the implementation of recipes in batch process operation are of significant industrial relevance. The paper describes a novel control-relevant formulation of the excessive liquid rise problem for a two-phase batch reactor subject to recipe uncertainties. The control simulations are carried out using a dedicated NMPC and optimization software toolbox Optcon which implements state of the art technologies. The open-loop optimal control problem is computed using the multipleshooting technique and the arising non-linear programming problem is solved using a sequential quadratic programming (SQP) algorithm tailored for large scale problems, based on the freeware optimization environment HQP. The fast response of the NMPC controller is guaranteed by the initial value embedding and real time iteration technologies. It is concluded that the OptCon implementation allows small sampling times and the controller is able to maintain safe and optimal operation conditions, with good control performance despite significant uncertainties in the implementation of the batch recipe.
\end{abstract}

Keywords:

Batch; Multiphase reactors; Process control; Multiphase flow; Optimization; Dynamic simulation;

\footnotetext{
* On research stay at the Loughborough University, U.K.

${ }^{1}$ Corresponding author. Tel: +41 44 6334486; fax: +41 446321189.

E-mail address: levente.simon@chem.ethz.ch (L. L. Simon)
} 


\section{Introduction}

Reactor or evaporator content swelling phenomena can lead to significant productivity losses if this phenomenon is not considered during process operation and is regarded as a reactor productivity and safety problem. Reactor content swelling occurs when the vessel content level rises due to a gas or vapor stream that passes through the liquid (Figure 1). Vapor flow occurs in a reactor when the reaction produces a gas phase product or during direct steam heating when some of the steam does not condense and disengages to the top of the vessel.

As a result of the swelling phenomena reaction mass enters the pipes and the condensers connected to the reactor. As a consequence of such undesired events reactor shut-down is mandatory and production time is lost for cleaning operations. The pipe and condenser cleaning is carried out by charging solvent which is evaporated and condensed for a certain time (refluxing conditions). The off-line optimal temperature control of batch reactors with regard to swelling was subject of investigation by Simon et al. (Simon et al., 2008a). Similarly to the batch reactors, reboiler liquid swelling may also occur during the operation of low pressure batch distillation processes (Simon et al., 2008b).

This work aims to implement an on-line model based level control strategy, which considers reaction content swelling. The on-line strategy is required to accommodate the reaction rate disturbances which arise due to catalyst dosing uncertainties (catalyst mass and feed time). Since the advent of dynamic matrix control (DMC), model predictive control (MPC) has been the most popular advanced control strategy in the chemical industries (Morari and Lee, 1997). Linear MPC has been heralded as a major advance in industrial process control (Richalet et al., 1978). However, due to their nonstationary and highly nonlinear nature, linear model based control usually cannot provide satisfactory performance in the case of complex batch processes (Qin and Badgwell, 2003). Nonlinear model predictive control (NMPC) reformulates the MPC problem based on nonlinear process models, providing the advantage to cope inherently with process nonlinearities (Allgower et al., 2004; Findeisen et al., 2007) characteristic to batch systems. Recent developments in the field of real-time optimization use the feedback information to adapt the constraints of the optimization problem instead of updating the model parameters (Chachuat et al., 2008). On-line optimization and integration of extended Kalman filter based estimation was shown to be an effective way to increase productivity of exothermic batch reactors (Arpornwichanop et al., 2005). Recent predictive control formulations that explicitly account for process 
nonlinearities and do not require the traditional assumption of initial feasibility of the optimization problem are presented by Mahmood and Mhaskar (2008) and Mhaskar et al. (2005). Zavala and Biegler (2009) proposed the advanced-step NMPC controller to reduce the on-line computational effort via the reformulation of the NMPC problem. New challenges related to the optimal transition from batch to continuous processing of bio-reactors, are handled using a non-linear model predictive controller to ensure the constraints satisfaction and performance targets (Mhaskar and Aumi, 2007). Robust formulations that incorporate parameter uncertainties in the control problem formulation are also available (Giovanini and Grimble, 2004; Nagy and Braatz, 2003).

The presented paper illustrates the benefits of the application of an efficient on-line optimizing non-linear model based control approach which uses the multiple shooting optimization algorithm. The optimization is implemented in a user-friendly software package, OptCon (Nagy et al., 2004; Nagy et al., 2007).

\section{Process operation and models}

The system considered in this study is based on a proprietary industrial batch process, for which the model has been developed and identified. The catalyst used in the chemical reaction decomposes in the reaction mixture; therefore it is fed several times during the process operation. The first feeding takes place at the beginning of the operation, later the catalyst shots are added as the reaction rate decreases. This type of process operation is often used in the industrial practice. The process is characterized by significant uncertainties in the kinetic constants and in the addition time of the catalyst. Figure 2 shows the experimental reaction rate measurements (normalized data) from the real industrial plant, in the case of repeated application of the same operating recipe with two consecutive catalyst dosing. The significant bath-to-batch variation may lead to safety problems and sub-optimal operation.

The process operation can be optimized off-line by calculating an optimal pressure profile in function of the catalyst dosage time, dosed mass and purity. However the off-line calculated optimal pressure profile does not ensure safe operation in the case of disturbances in the catalyst feeding policy. Instead an on-line strategy is needed to adjust the pressure profile during the operation considering the unknown disturbances. The control strategy used here is based on the nonlinear model predictive control (NMPC) framework for batch processes.

\subsection{Batch reactor modeling}


Four equilibrium reactions in series take place in the liquid phase and a catalyst is used in dissolved form. The reaction scheme is as follows:

$$
\begin{aligned}
& \mathrm{A}+\mathrm{B} \rightleftarrows \mathrm{C}+\mathrm{D} \\
& \mathrm{B}+\mathrm{C} \rightleftarrows \mathrm{E}+\mathrm{D} \\
& \mathrm{B}+\mathrm{E} \rightleftarrows \mathrm{F}+\mathrm{D} \\
& \mathrm{B}+\mathrm{F} \rightleftarrows \mathrm{P}+\mathrm{D} \\
& \mathrm{B}+\text { Catalyst } \rightarrow \mathrm{W}
\end{aligned}
$$

Raw materials are component $\mathrm{A}$ and $\mathrm{B}$; components $\mathrm{C}, \mathrm{E}, \mathrm{F}$ are intermediates, $\mathrm{P}$ is the desired product and $\mathrm{W}$ is the decomposed catalyst. The goal of the process operation is to remove the co-product $\mathrm{D}$ from the liquid phase as fast as possible to shift the equilibrium reactions to the product side. Product $\mathrm{D}$ is in vapour phase at the temperature and pressure conditions in the reactor, and the production of the co-product D creates a vapour flow that travels to the reaction mass surface and produces a certain void fraction in the liquid mass. The extent of the void fraction is dependent on the liquid properties and vapour hold-up in liquid phase which in turn are dependent on the vapour flow rate and implicitly on the formation rate of gas co-product $\mathrm{D}$.

By operating at low pressure conditions, the amount of D in liquid phase is minimized, thus the reverse reactions are not significant. Therefore, the kinetic model is based only on forward reactions which ensure a safety back-off in the model. The true reaction rates will never be faster than the forward reactions.

In order to model the forward reactions the Arrhenius formulation is implemented, using a reference reaction constant determined at a reference temperature (Bunce et al., 1988):

$$
\begin{aligned}
& r_{R}^{i}=k_{r e f}^{i} \exp \left\{-\frac{E_{A, i}}{R}\left(\frac{1}{T_{r}}-\frac{1}{T_{r e f}}\right)\right\} \frac{n_{C a t} \cdot n_{B} \cdot n_{X}}{V^{3}} \\
& r_{C a t}=k_{C a t} \exp \left\{-\frac{E_{C a t}}{R}\left(\frac{1}{T_{r}}-\frac{1}{T_{r e f}}\right)\right\} \frac{n_{C a t} \cdot n_{B}}{V^{2}}
\end{aligned}
$$


where $i$ is the $i$ th reaction step, $r_{R}^{i}$ is the $i$ th reaction rate to the right-hand side $\left[\mathrm{kmol} /\left(\mathrm{m}^{3} \mathrm{~s}\right)\right]$, $r_{C a t}$ is the catalyst deactivation rate, $k_{r e f}^{i}$ are the corresponding rate constants at reference temperature $\left[\mathrm{m}^{6} /\left(\mathrm{kmol}^{2} \mathrm{~s}\right)\right], k_{C a t}$ is the catalyst deactivation rate constant at reference temperature $\left[\mathrm{m}^{3} /(\mathrm{kmol} \mathrm{s})\right], E_{A, i}$ the activation energies $[\mathrm{kJ} / \mathrm{kmol}], E_{C a t}$ the catalyst deactivation reaction activation energy $[\mathrm{kJ} / \mathrm{kmol}], T_{r}$ and $T_{r e f}$ are the current and reference temperature $[\mathrm{K}], R$ is the gas constant $[\mathrm{kJ} /(\mathrm{kmol} \mathrm{K})], n_{B}$ is the mole number of component $\mathrm{B}$ [kmol], $n_{C a t}$ is the catalyst mol number [kmol], $n_{X}$ represents $n_{A}, n_{C}, n_{E}$ and $n_{F}[\mathrm{kmol}]$, respectively, and $V$ is the volume of the reaction mass $\left[\mathrm{m}^{3}\right]$. During the reaction the volume changes significantly, therefore $V$ is a variable in the model. The reaction volume is not constant due to two factors: on one hand there is the removal of by-product $\mathrm{D}$ and on the other hand the density of the mixture changes. These two effects contribute each with about $10 \%$ volume change. The reaction volume at any time is calculated as a function of the densities and masses of all components in the mixture thus accounting for the removal of coproduct $\mathrm{D}$ and the change in composition. The resulting component mass balances for the liquid phase are as follows:

$$
\begin{aligned}
& \frac{d n_{A}}{d t}=-r_{R}{ }^{1} V \\
& \frac{d n_{B}}{d t}=\left(-r_{R}{ }^{1}-r_{R}{ }^{2}-r_{R}{ }^{3}-r_{R}{ }^{4}-r_{\text {Cat }}\right) V \\
& \frac{d n_{C}}{d t}=\left(r_{R}{ }^{1}-r_{R}{ }^{2}\right) V \\
& \frac{d n_{D}}{d t}=\left(r_{R}{ }^{1}+r_{R}{ }^{2}+r_{R}{ }^{3}+r_{R}{ }^{4}\right) V \\
& \frac{d n_{E}}{d t}=\left(r_{R}{ }^{2}-r_{R}{ }^{3}\right) V \\
& \frac{d n_{F}}{d t}=\left(r_{R}{ }^{3}-r_{R}{ }^{4}\right) V \\
& \frac{d n_{P}}{d t}=r_{R}^{4} V \\
& \frac{d n_{\text {Cat }}}{d t}=-r_{\text {Cat }} V+F_{\text {cat }}
\end{aligned}
$$

where $F_{c a t}$ is the catalyst dosing rate $[\mathrm{kmol} / \mathrm{s}]$.

\subsection{Void fraction modeling}


In order to describe the effect of liquid swelling the pool void fraction, is used. The swelled height $H[\mathrm{~m}]$ in terms of the average pool void fraction $\bar{\alpha}$ and the height of the resting liquid $H_{0}[\mathrm{~m}]$ is given by the following equation:

$$
H=\frac{H_{0}}{1-\bar{\alpha}}
$$

Wilson et al. determined the void fraction $\bar{\alpha}$ by bubbling steam through water in a pressurized vessel in the 20-40 bar pressure range (Wilson et al., 1961). Their proposed empirical correlation is presented below:

$\bar{\alpha}=K\left(\frac{\rho_{V}}{\rho_{L}-\rho_{V}}\right)^{0.17}\left[\sqrt{\frac{\sigma}{g\left(\rho_{L}-\rho_{V}\right)}} / D_{r e b}\right]^{0.1}\left[j_{V} /\left(g \sqrt{\frac{\sigma}{g\left(\rho_{L}-\rho_{V}\right)}}\right)^{0.5}\right]^{f}$

with

$K=0.68, f=0.62$ for

$$
j_{V} /\left(g \sqrt{\frac{\sigma}{g\left(\rho_{L}-\rho_{V}\right)}}\right)^{0.5}<2
$$

$K=0.88, f=0.40$ for

$$
j_{V} /\left(g \sqrt{\frac{\sigma}{g\left(\rho_{L}-\rho_{V}\right)}}\right)^{0.5} \geq 2
$$

where $\rho_{L}$ and $\rho_{V}\left[\mathrm{~kg} / \mathrm{m}^{3}\right]$ are the liquid and vapor densities, $\sigma[\mathrm{N} / \mathrm{m}]$ is the surface tension, $g$ $\left[\mathrm{m} / \mathrm{s}^{2}\right]$ is the gravitational acceleration, $D_{r e b}[\mathrm{~m}]$ is the reboiler vessel diameter and $j_{V}[\mathrm{~m} / \mathrm{s}]$ is the superficial vapor velocity. To our knowledge void fraction correlations valid under vacuum conditions are not available in the literature, therefore a set of experiments within 0.1 and 0.8 bar was carried out. It was found that at 0.1 bar, the Wilson model predicts correctly the void fraction values up to $10-15 \%$ (Simon and Hungerbuhler, 2009). The comparisons of the void fraction predictions as a function of the gas flow rate using the four hydrodynamic models is discussed in Simon et al. (Simon et al., 2008b). It is concluded that, at 1 bar pressure, the Wilson, Churn turbulent (Fisher et al., 1992) and Sterman models (Sterman, 1958) show similar behavior, and the Kataoka-Ishii model (Kataoka and Ishii, 1987) predicts larger void fractions. The four hydrodynamic models were compared at 0.25 bar pressure as well; the comparison results show that the Wilson and Churn turbulent model predictions are similar, while the Sterman and Katoka-Ishii models predict larger void fractions. It is interesting to note that the Wilson model was successfully extrapolated by Wiss et al. (Wiss 
et al., 1993) (without refitting the model parameters) from about 20-40 bar pressure to 1 bar. In conclusion, our experimental results have proved that the Wilson model is a good choice for pool boiling modeling under vacuum conditions, thus it is used in the model applied in the current calculations.

3. On-line optimizing control for swelling constrained batch reactor

The on-line optimizing control for a model represented by a generic ordinary-differential equation (ODE) system can be expressed as follows:

$$
\begin{aligned}
& \dot{x}(t)=f(x(t), u(t)) \\
& y(t)=g(x(t), u(t))
\end{aligned}
$$

subject to the input, state and output constraints

$$
u(t) \in U, x(t) \in X, y(t) \in Y
$$

where $x(t)$ is the $n_{x}$ vector of states, $u(t)$ is the $n_{u}$ set of input vector trajectories and $y(t)$ is the $n_{y}$ vector of output variables. The sets $X$ and $Y$ are closed subsets of $\mathfrak{R}^{n_{x}}$ and $\mathfrak{R}^{n_{y}}$, respectively and the set $U$ is a compact subset of $\mathfrak{R}^{n_{u}}$. If we suppose that the full state $x$ can be measured, then in the batch NMPC (Nagy et al., 2004; Nagy et al., 2007) the control input applied to the system in the interval $\left[t_{k}, t_{f}\right]$ is given by the repeated solution of the finite horizon optimal control problem given by:

$$
\begin{array}{cl}
\min _{\bar{u}(\cdot)}\left\{M\left(x\left(t_{f}\right)\right)+\int_{t_{k}}^{t_{F}} \Psi(\bar{x}(t), \bar{u}(t)) d t\right\} \\
\text { s.t. } \quad \dot{\bar{x}}(t)=f\left(\bar{x}(t), \bar{u}(t), \bar{x}\left(t_{k}\right)=x\left(t_{k}\right)\right) \\
\\
\bar{u}(t) \in U, \forall t \in\left[t_{k}, t_{f}\right] \\
\quad \bar{x}(t) \in X, \forall t \in\left[t_{k}, t_{f}\right] \\
\bar{y}(t) \in Y, \forall t \in\left[t_{k}, t_{f}\right]
\end{array}
$$

where the objective function has the generic form, which consists of the end-point objective $(M)$ and a path term $(\Psi), t_{k}$ denotes the sampling instance, $t_{f}$ is the batch time and $t_{F} \leq t_{f}$ is 
the prediction horizon for the running term. Although in the case of typical batch NMPC only the end-point objective is considered, based on the nature of the control objective in practical cases often either one or both terms may be incorporated in the actual objective function. When $t_{F}=t_{f}$ the optimization is performed on a shrinking horizon, whereas if $t_{F} \leq t_{f}$ initially the problem is solved on a combination of shrinking and moving horizon until $t_{k}+t_{F}<t_{f}$ after which on shrinking horizon. The bar in the optimization problem denotes the predicted variables, i.e. $\bar{x}$ denotes the solution of the system driven by the input $\bar{u}$ with the initial condition $x\left(t_{k}\right)$. Even if in the case of shrinking horizon NMPC in the nominal case the real state $x$ of the system coincides with the predicted state $\bar{x}$, it is necessary to make a distinction between the two due to differences which occur due to uncertainties in model parameters, inputs and disturbances.

The repeated optimization problem is solved by formulating a discrete form, that can be handled by conventional solvers (Biegler and Rawlings, 1991). The batch time $t \in\left[0, t_{f}\right]$ is divided into $N$ equally spaced time intervals $\Delta t$ (stages), with discrete time steps $t_{k}=k \Delta t$, and $k=0,1, \ldots, N$. The main idea of the shrinking horizon on-line control algorithm (batch NMPC) is summarized as follows:

1. with known initial conditions, discretize batch time in $N$ intervals;

2. optimize property at the end of the batch;

3. implement calculated input for the first control interval;

4. initialize optimization with states taken at the end of time interval $k$;

5. re-optimize property at the end of the batch, having $N-1$ decision variables in the optimal control problem;

6. implement the first control input;

7. go back to step 4, and repeat until the end of batch.

For the solution of the optimization problem a specially tailored NMPC tool - OptCon - was developed that includes a number of desirable features (Nagy et al., 2004). In particular, the NMPC is based on first-principles or grey box models, and the problem setup can be done in Matlab. The NMPC approach is based on a large-scale NLP solver (HQP) (Franke et al., -, Nagy et al., 2007) which offers an efficient optimization environment, based on multiple shooting algorithm (Bock and Plitt, 1984; Diehl et al., 2002), that divides the optimization horizon into a number of subintervals (stages) with local control parameterizations. The 
differential equations and cost on these intervals are integrated independently during each optimization iteration. The continuity/consistency of the state trajectory at the end of the optimization is enforced by adding consistency constraints to the nonlinear programming problem. The NMPC tool OptCon provides an efficient environment for rapid prototyping of NMPC strategies even in an industrial environment and has been successfully used in a variety of practical applications (Nagy et al., 2007).

\section{Results and discussion}

\subsection{Open-loop optimal control of the swelling constrained batch reactor}

The most widely used optimization method to improve the batch processes is the formulation and solution of an optimal control problem (Bonvin, 1998). The open-loop optimisation problem corresponds to the first optimization step in the batch NMPC algorithm described in the previous section. In this approach the objective is to improve an end-point criteria by calculating a time variant input variable profile (temperature, pressure, feed rate).

Since the reactions are chemical equilibrium limited it is important that the pressure in the reactor is the lowest possible. However, minimum reactor pressure yields maximum gas volume and eventually maximum liquid level, thus this limitation has to be taken into account during the calculation of the pressure profile. Although it is plausible to control the cause of the vapor formation (reaction kinetics) using the temperature, in practice this is not possible due to the slow dynamics of temperature change in large scale reactors. Instead, from practical point of view, it is more feasible to control the true level by means of the pressure. For this case study the temperature ramp is set to $1 \mathrm{C} / \mathrm{min}$.

The optimization of the batch reactor with regard to swelling can be regarded as a problem to determine the pressure profile which will not cause the level to rise over a maximum value. The objective function is to maximize the component $B$ depletion or to minimize the content of component $B$ at final time, the control variable is the pressure, and the final simulation time is fixed to $40 \mathrm{~min}$. The inequality path constraint is the formation rate of co-product $\mathrm{D}$ converted in true reactor content level. The optimal control problem for this process is formulated as follows:

$$
M=\min _{P(t)} \int_{0}^{t_{f}} P(t) d t
$$


Subject to:

$$
\begin{aligned}
& g\left(\text { Rdae }\left(\dot{x}, x, T, v_{R}, c_{R}^{\text {Model }}\right), \operatorname{Hydro}\left(\dot{x}, T, P, v_{R}, c_{\text {Hydro }}^{\text {Model }}\right), t_{f}, t\right)=0 \\
& H_{\max }-H_{r}(t) \geq 0 \\
& P_{\max }-P(t) \geq 0 \\
& P(t)-P_{\min } \geq 0
\end{aligned}
$$

where $t_{f}$ is the final time (end of the optimization) at which it is considered that swelling cannot occur anymore (not yet the end of the reaction), $P$ is the pressure proposed by the optimizer, Rdae is the kinetic model, $v_{R}$ is the vector of reaction mass specific constants, $c_{R}^{\text {Model }}$ is the vector of reaction model specific parameters, Hydro is the hydrodynamic model, $c_{H y d r o}^{\text {Model }}$ is the vector of hydrodynamic model specific constants and parameters, $H_{\max }(2.4 \mathrm{~m})$ and $H_{r}(t)$ are the maximum level and calculated reactor content level, respectively, $P_{\max }(1$ bar) and $P_{\min }(0.075$ bar $)$ are the maximum and the minimum pressures, respectively. The objective function is expressed as the integral of the pressure profile over the batch time. This formulation guaranties that the maximum level is not exceeded while the pressure is kept at the minimum possible level, which indirectly maximizes productivity. Figure 3 shows (a) the open-loop optimal pressure profile and (b) the calculated reactor level. The pressure increases due to the catalyst feeding procedure which happens during short periods at time steps 0,15 , 25, 35 minutes during which the reaction rate increases. However, during the operation of the batch reactor there is an uncertainty regarding the catalyst mass and dosing time which causes significant level increase and sub-optimal operation. The Figure $3 \mathrm{~b}$ shows the true reactor level in case when the catalyst is dosed 3 minutes earlier compared to the recipe and also considering that $20 \%$ more mass is added. The sampling time is $20 \mathrm{~s}$.

\subsection{On-line re-optimizing control of the swelling constrained batch reactor}

In order to cope with the disturbance of the chemical system a shrinking horizon, reoptimizing model based control strategy is implemented. The optimal control problem defined by equations (25)-(29) is solved repeatedly considering a state feedback formulation. This assumption is used since in practice the concentrations (states) are measurable with spectroscopy based techniques such as infrared (IR) spectroscopy. The measured spectra are previously calibrated to known concentration samples using partial-least squares (PLS) 
models. The spectral peak position and intensity are chemistry dependent and are used in the industrial environment.

The level control results for the case when the catalyst is fed with 3 minutes earlier and with $20 \%$ more mass than specified by the recipe is presented in Figure 4. Due to the small sampling time the controller is able to accommodate the change of vapor rate and it maintains the level at the maximum level. In this case the information contained in the original recipe is not used by the model during the open-loop optimization, since the catalyst dosing is expected later.

The next set of simulations is concerned with the case when the catalyst is fed delayed and with $20 \%$ more mass as set in the recipe. Figure 5 presents the case when the catalyst feed is delayed with 3 minutes. Similarly to the previous case the level control is good despite the disturbance in the recipe.

In order to analyze the effect of the recipe information on the process control behavior a scenario was simulated in which the catalyst feeding time is delayed by 30 seconds (the catalyst feeding takes 1 minute). This way it is expected that the controller has some information about the dosing time in the open-loop optimization. This expectation is confirmed in Figure 6b, where we observe that the level is lower within the first sampling time.

In order to verify the influence of the model-plant mismatch, a slower, thus conservative model was used. In the kinetic model the activation energies were increased by $30 \%$ and the prediction of the hydrodynamic model was decreased by $30 \%$. This way the true level height is under predicted. The simulation results considering early catalyst feeding ( 3 minutes sooner) and the model discussed above are presented in Figure 7. An offset compared to the maximum level can be observed, which can be decreased by running the optimization problems with the level constrained at $2.3 \mathrm{~m}$. Using a model which under predicts the level the optimizer will try to lower the pressure to a higher extent compared to the prediction which is based on an accurate model. For low pressures the effect of plant-model mismatch is controlled by the lower bound on the pressure which is the control variable. 
We would like to highlight the importance of the real-time feasibility of the calculations, especially during the catalyst feeding period when excessive level increase may occur. As shown in the results presented above using small sampling time the control performance is good. There may be cases when the NMPC strategy may not allow the implementation of small sampling time control actions. In these cases the open-loop formulation of the NMPC control problem, according to which the NMPC control actions are applied in an open-loop way between the NMPC sampling instances, may not provide acceptable results. During the open-loop control period a disturbance may lead to the rapid rise of the reactor level and hence to the violation of safety constraint. In these cases a closed-loop NMPC can be implemented. According to this strategy the optimization repeatedly finds a feedback law rather than an open-loop profile. The simplest control law is a linear output feedback level controller,

$$
P(t)=K(k)\left(H_{s e t}-H(t)\right)
$$

where, $K(k)$ is the dynamic controller gain which is fixed on a discretization interval and is the result of the closed-loop NMPC optimization problem (Nagy and Braatz, 2004). 


\section{Conclusions}

The paper presents an on-line optimizing batch process control strategy with respect to excessive liquid rise. The on-line strategy is required to accommodate the reaction rate disturbances which arise due to catalyst dosing uncertainties (variations in catalyst mass and feeding time). The control simulations are carried out using a dedicated NMPC and optimization software toolbox Optcon which implements state of the art technologies. The open-loop optimal control problem is computed using the multiple-shooting technique and the arising non-linear programming problem is solved using a sequential quadratic programming (SQP) algorithm tailored for large scale problems, based on the freeware optimization environment HQP. The fast response of the NMPC controller is guaranteed by the initial value embedding and real time iteration technologies. This efficient NMPC scheme allows the implementation of control actions with small sampling time, which is an important aspect in this application, which may exhibit very rapid rise in level due to swelling. The simulation results obtained using the model fitted to industrial experimental data indicate that the on-line model based control is able to keep the reactor level within safety operation constraints, without causing excessive liquid swelling or sub-optimal operation even in the case of significant deviations from the master recipe.

\section{Acknowledgments}

The financial support from Ciba Specialty Chemicals, Switzerland is greatly acknowledged. 
List of symbols

Latin symbols

\begin{tabular}{|c|c|}
\hline$c_{\text {Hydro }}^{\text {Model }}$ & vector of hydrodynamic model specific constants and parameters \\
\hline$c_{R}^{M o d e l}$ & vector of reactor model specific constants and parameters \\
\hline$d n$ & vector of accumulation or consumption rates of all components, $[\mathrm{kmol} / \mathrm{s}]$ \\
\hline$E_{\text {Cat }}$ & catalyst deactivation reaction activation energy, $[\mathrm{kJ} / \mathrm{kmol}]$ \\
\hline$E_{A i}$ & activation energies, $[\mathrm{kJ} / \mathrm{kmol}]$ \\
\hline$F_{\text {Cat }}$ & catalyst dosing rate, $[\mathrm{kmol} / \mathrm{s}]$ \\
\hline$G$ & set of dynamic equations and set of equality constraints \\
\hline$H$ & swelled vessel height, $[\mathrm{m}]$ \\
\hline$H_{0}$ & height of the resting liquid, $[\mathrm{m}]$ \\
\hline$H_{\max }$ & maximum reactor level, $[\mathrm{m}]$ \\
\hline Hydro & hydrodynamic model \\
\hline$j_{g}$ & vapor superficial velocity, $[\mathrm{m} / \mathrm{s}]$ \\
\hline$K$ & dynamic controller gain \\
\hline$k$ & discretization interval \\
\hline$k_{\text {Cat }}$ & rate constant at reference temperature, $\left[\mathrm{m}^{3} /(\mathrm{kmol} \mathrm{s})\right]$ \\
\hline$k_{r e f}^{i}$ & rate constants at reference temperature, $\left[\mathrm{m}^{6} /\left(\mathrm{kmol}^{2} \mathrm{~s}\right)\right]$ \\
\hline$M$ & objective function \\
\hline $\begin{array}{l}n_{A}, n_{B}, n_{C}, n_{D}, n_{E} \\
n_{F}, n_{P}, n_{W}\end{array}$ & mole number of component A, B, C, D, E, F, P, W [kmol] \\
\hline$N$ & number of intervals \\
\hline$R$ & gas constant, $\left[\mathrm{kJ} / \mathrm{kmol}{ }^{\circ} \mathrm{C}\right]$ \\
\hline$r_{\text {Cat }}$ & catalyst deactivation rate, $\left[\mathrm{kmol} /\left(\mathrm{m}^{3} \mathrm{~s}\right)\right]$ \\
\hline $\mathfrak{R}^{n}$ & set of inputs, states, outputs \\
\hline$r_{R}^{i}$ & $\mathrm{i}^{\text {th }}$ reaction rate to the right-hand side, $\left[\mathrm{kmol} /\left(\mathrm{m}^{3} \mathrm{~s}\right)\right]$ \\
\hline$T$ & reactor temperature, $\left[{ }^{\circ} \mathrm{C}\right]$ \\
\hline$T_{r e f}$ & reference temperature, $\left[{ }^{\circ} \mathrm{C}\right]$ \\
\hline$t_{f}$ & final time, $[\mathrm{s}]$ \\
\hline$U_{\infty}$ & characteristic bubble rise velocity, $[\mathrm{m} / \mathrm{s}]$ \\
\hline$u$ & input \\
\hline$V$ & reactor mass volume, $\left[\mathrm{m}^{3}\right]$ \\
\hline$V_{g}$ & average local slip velocity, $[\mathrm{m} / \mathrm{s}]$ \\
\hline $\mathrm{x}$ & states \\
\hline $\bar{x}$ & predicted state \\
\hline
\end{tabular}


output

Greek letters

$\bar{\alpha}$

$\rho_{L}$

$\rho_{V}$

$\Psi$

$\sigma$

\section{Superscript}

2

3

4

\section{Subscript}

Cat

$i$

ref pool void fraction, [-]

liquid density, $[\mathrm{kg} / \mathrm{m} 3]$

vapor density, $[\mathrm{kg} / \mathrm{m} 3]$

path term

surface tension, $[\mathrm{N} / \mathrm{m}]$

\section{Reaction 1}

Reaction 2

Reaction 3

Reaction 4

catalyst

ith reaction step

reference 
References

Allgower, F., Findeisen, R., Nagy, Z. K., (2004). Nonlinear Model Predictive Control: From Theory to Application. Journal of the Chinese Institute of Chemical Engineers, 35, 299-315. Arpornwichanop, A., Kittisupakorn, A., Mujtaba, I. M., (2005). On-Line Dynamic Optimization and Control Strategy for Improving the Performance of Batch Reactors. Chemical Engineering and Processing, 44, 101-114.

Biegler, L., Rawlings, J., Optimization Approaches to Nonlinear Model Predictive Control, In Conference of Chemical Process Control, South Padre Island, TX, 1991.

Bock, H. G., Plitt, K. J., A Multiple Shooting Algorithm for Direct Solution of Optimal Control Problems, In Proceedings 9th IFAC World Congress, Budapest, 1984.

Bonvin, D., (1998). Optimal Operation of Batch Reactors - a Personal View. Journal of Process Control, 8, 355-368.

Bunce, N. J., Forber, C. L., McInnes, C., Hutson, J. M., (1988). Single-Step Methods for Calculating Activation Parameters from Raw Kinetic Data. Journal of the Chemical Society, Perkin Transactions 2: Physical Organic Chemistry, 3, 363-368.

Chachuat, B., Marchetti, A., Bonvin, D., (2008). Process Optimization Via Constraints Adaptation. Journal of Process Control, 18, 244-257.

Diehl, M., Bock, H. G., Schloder, J. P., Findeisen, R., Nagy, Z., Allgower, F., (2002). RealTime Optimization and Nonlinear Model Predictive Control of Processes Governed by Differential-Algebraic Equations. Journal of Process Control, 12, 577-585.

Findeisen, R., Allgoewer, F., Biegler, L., (2007). Assessment and Future Directions of Nonlinear Model Predictive Control. Lecture Notes in Control and Information Sciences. Springer Berlin: Heidelberg.

Fisher, H. G., Forrest, H. S., Grossel, S. S., Huff, J. E., Muller, A. R., Noronha, J. A., Shaw, D. A., Tilley, B. J., (1992). Emergency Relief System Design Using Diers Technology. American Institute of Chemical Engineers: New-York. http://hqp.sourceforge.net/, Hqp: A Solver for Nonlinearly Constrained Large-Scale Optimization. .

Giovanini, L., Grimble, M., (2004). Robust Predictive Feedback Control for Constrained Systems. International Journal of Control Automation and Systems, 2, 407-422.

Kataoka, I., Ishii, M., (1987). Drift Flux Model for Large Diameter Pipe and New Correlation for Pool Void Fraction. International Journal of Heat and Mass Transfer, 30, 1927-1939. Mahmood, M., Mhaskar, P., (2008). Enhanced Stability Regions for Model Predictive Control of Nonlinear Process Systems. AIChE Journal, 54, 1487-1498.

Mhaskar, P., Aumi, S., (2007). Transition from Batch to Continuous Operation in BioReactors: A Model Predictive Control Approach and Application. Canadian Journal of Chemical Engineering, 85, 416-423.

Mhaskar, P., El-Farra, N. H., Christofides, P. D., (2005). Predictive Control of Switched Nonlinear Systems with Scheduled Mode Transitions. Ieee Transactions on Automatic Control, 50, 1670-1680.

Morari, M., Lee, J. H., Model Predictive Control: Past, Present and Future., In PSE'97ESCAPE-7 Symposium, Trondheim, 1997.

Nagy, Z. K., Allgöwer, F., Franke, R., Frick, A., Mahn, B., Efficient Tool for Nonlinear Model Predictive Control of Batch Processes, In 12th Mediterranean Conference on Control and Automation MED'04, Kusadasi, Turkey, 2004.

Nagy, Z. K., Braatz, R. D., (2003). Robust Nonlinear Model Predictive Control of Batch Processes. AIChE Journal, 49, 1776-1786. 
Nagy, Z. K., Braatz, R. D., (2004). Open-Loop and Closed-Loop Robust Optimal Control of Batch Processes Using Distributional and Worst-Case Analysis. Journal of Process Control, 14, 411-422.

Nagy, Z. K., Mahn, B., Franke, R., Allgower, F., (2007). Evaluation Study of an Efficient

Output Feedback Nonlinear Model Predictive Control for Temperature Tracking in an

Industrial Batch Reactor. Control Engineering Practice, 15, 839-850.

Qin, S. J., Badgwell, T. A., (2003). A Survey of Industrial Model Predictive Control

Technology. Control Engineering Practice, 11, 733-764.

Richalet, J., Rault, A., Testud, J. L., Papon, J., (1978). Model Predictive Heuristic Control Applications to Industrial Processes. Automatica, 14, 413-428.

Simon, L. L., Hungerbuhler, K., (2009). Experimental Determination of Pool Void Fraction under Vacuum Conditions. Chemical Engineering \& Technology, 32.

Simon, L. L., Introvigne, M., Fischer, U., Hungerbuhler, K., (2008a). Batch Reactor Optimization under Liquid Swelling Safety Constraint. Chemical Engineering Science, 63, 770-781.

Simon, L. L., Kencse, H., Hungerbuhler, K., (2008b). Optimal Rectification Column, Reboiler Vessel, Connection Pipe Selection and Optimal Control of Batch Distillation Considering Hydraulic Limitations. Chemical Engineering and Processing: Process Intensification, in press.

Sterman, L. S., (1958). On the Theory of Steam Separation. Soviet Physics-Technical Physics, 3, 1440-1451.

Wilson, J. F., Grenda, R. J., Patterson, J. F., (1961). Steam Volume Fraction in Bubbling Two-Phase Mixture. Transactions of the American Nuclear Society, 4, 356-357.

Wiss, J., Stoessel, F., Kille, G., (1993). A Systematic Procedure for the Assessment of the Thermal Safety and for the Design of Chemical Processes at the Boiling-Point. Chimia, 47, 417-423.

Zavala, V. M., Biegler, L. T., (2009). The Advanced-Step Nmpc Controller: Optimality, Stability and Robustness. Automatica, 45, 86-93. 
Figures

Figure 1 Swelled liquid in a $100 \mathrm{~L}$ pilot plant vessel.

Figure 2 Change of the reaction rate in time for an existing batch process.

Figure 3 Open loop calculated pressure profile (a) and the corresponding reactor level (b), straight lines; dashed line shows the reactor level according to the disturbed conditions. Catalyst dosing at 15 min, 25 min and 35 min.

Figure 4. Pressure profile (a) and the corresponding reactor level for an early addition disturbance case. Catalyst dosing at $12 \mathrm{~min}, 22 \mathrm{~min}$ and $32 \mathrm{~min}$.

Figure 5. Pressure profile (a) and the corresponding reactor level for a late addition (with $3 \mathrm{~min}$ ) disturbance case. Catalyst dosing at $18 \mathrm{~min}, 28 \mathrm{~min}$ and $38 \mathrm{~min}$.

Figure 6. Pressure profile (a) and the corresponding reactor level for a late addition (with $30 \mathrm{~s}$ ) disturbance case (b). Catalyst dosing at $15.5 \mathrm{~min}, \mathbf{2 5 . 5} \mathrm{min}$ and $35.5 \mathrm{~min}$.

Figure 7. Pressure profile (a) and the corresponding reactor level (b) for an early addition (with 3 min) disturbance case, using a model with significant model-pant mismatch. Catalyst dosing at 12 min, 22 min and $32 \mathrm{~min}$. 


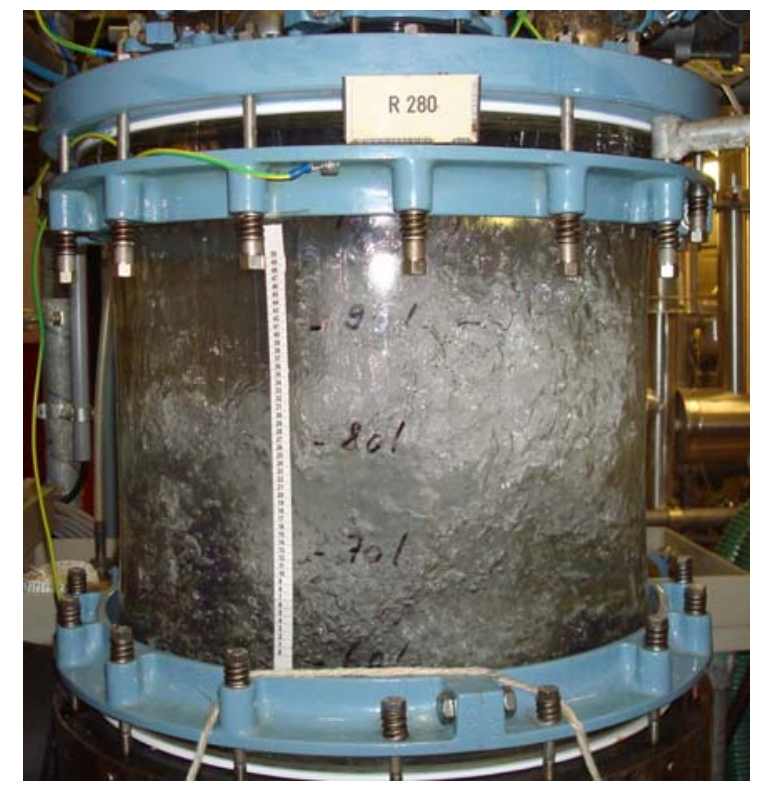

Figure 1 Swelled liquid in a $100 \mathrm{~L}$ pilot plant vessel. 


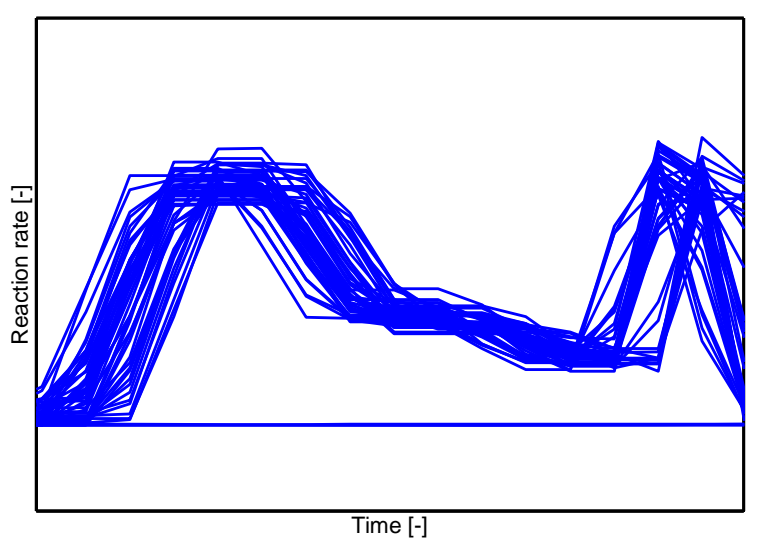

Figure 2 Change of the reaction rate in time for an existing batch process.

20 


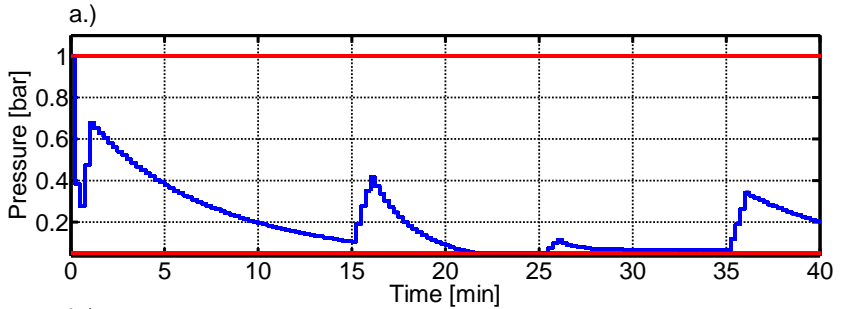

b.)

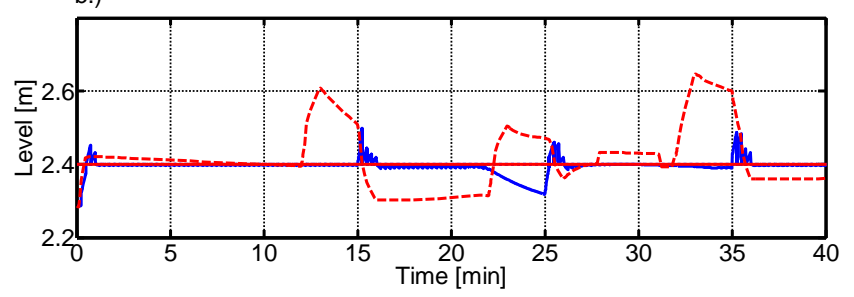

Figure 3 Open loop calculated pressure profile (a) and the corresponding reactor level (b), straight lines; dashed line shows the reactor level according to the disturbed conditions. Catalyst dosing at 15 min, 25 min and 35 min. 

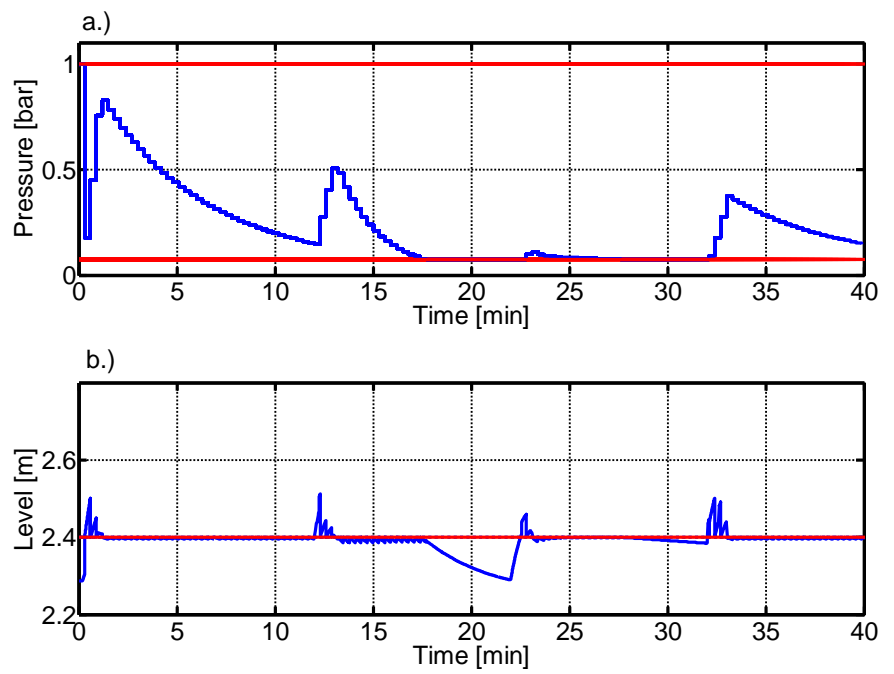

Figure 4. Pressure profile (a) and the corresponding reactor level for an early addition disturbance case. Catalyst dosing at $12 \mathrm{~min}, 22 \mathrm{~min}$ and $32 \mathrm{~min}$. 
a.)

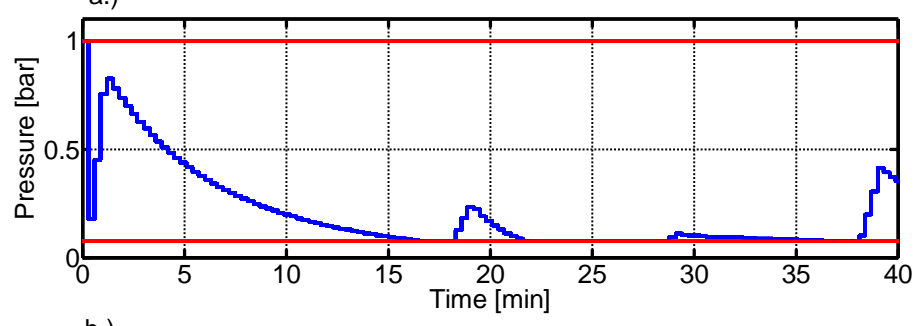

b.)

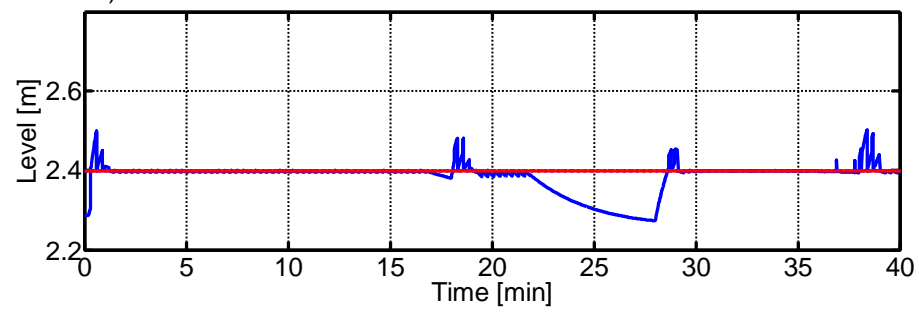

Figure 5. Pressure profile (a) and the corresponding reactor level for a late addition (with 3 min) disturbance case. Catalyst dosing at $18 \mathrm{~min}, 28 \mathrm{~min}$ and $38 \mathrm{~min}$. 

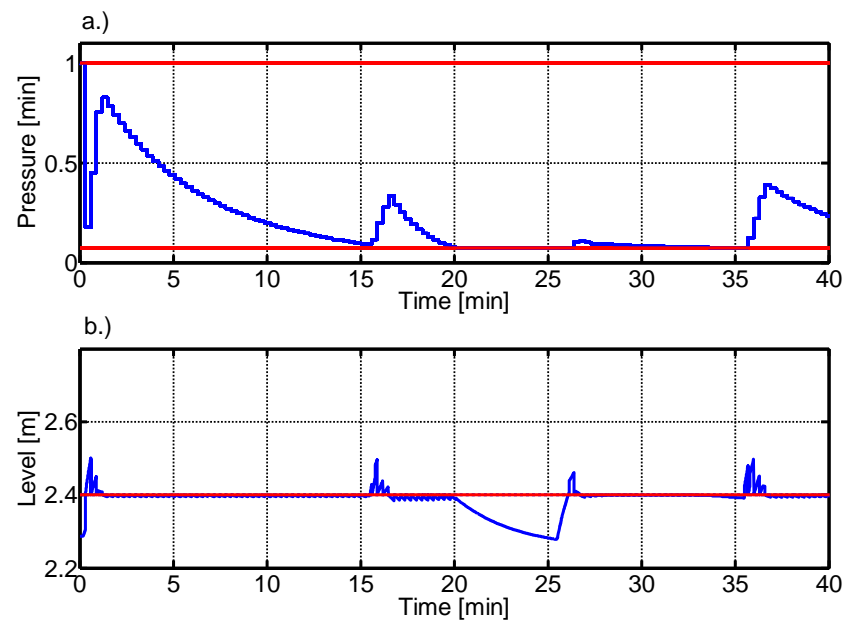

Figure 6. Pressure profile (a) and the corresponding reactor level for a late addition (with $30 \mathrm{~s}$ ) disturbance case (b). Catalyst dosing at $15.5 \mathrm{~min}, \mathbf{2 5 . 5} \mathrm{min}$ and $35.5 \mathrm{~min}$. 


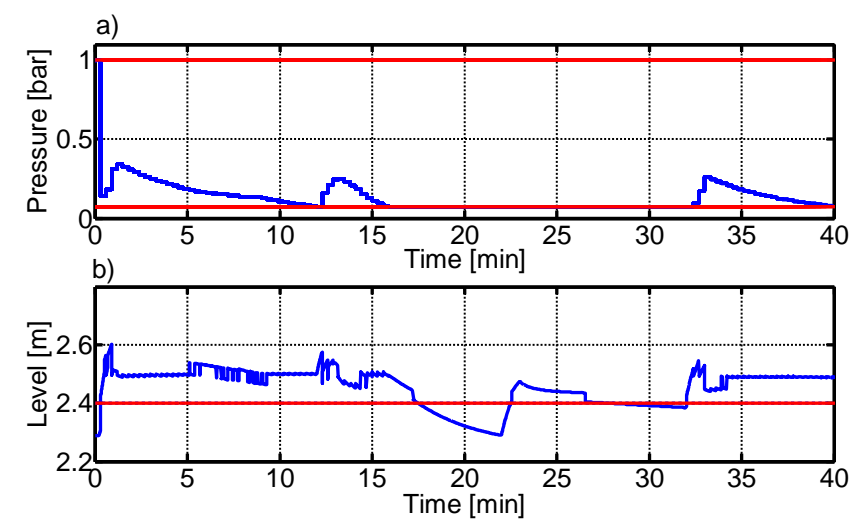

Figure 7. Pressure profile (a) and the corresponding reactor level (b) for an early addition (with 3 min) disturbance case, using a model with significant model-pant mismatch. Catalyst dosing at $12 \mathrm{~min}, 22 \mathrm{~min}$ and $32 \mathrm{~min}$. 